\title{
Principal Gabor Filters for Face Recognition
}

\author{
Vitomir Štruc, Rok Gajšek and Nikola Pavešić
}

\begin{abstract}
Gabor filters have proven themselves to be a powerful tool for facial feature extraction. An abundance of recognition techniques presented in the literature exploits these filters to achieve robust face recognition. However, while exhibiting desirable properties, such as orientational selectivity or spatial locality, Gabor filters have also some shortcomings which crucially affect the characteristics and size of the Gabor representation of a given face pattern. Amongst these shortcomings the fact that the filters are not orthogonal one to another and are, hence, correlated is probably the most important. This makes the information contained in the Gabor face representation redundant and also affects the size of the representation. To overcome this problem we propose in this paper to employ orthonormal linear combinations of the original Gabor filters rather than the filters themselves for deriving the Gabor face representation. The filters, named principal Gabor filters for the fact that they are computed by means of principal component analysis, are assessed in face recognition experiments performed on the XM2VTS and YaleB databases, where encouraging results are achieved.
\end{abstract}

\section{INTRODUCTION}

Deriving a discriminative and compact representation of a face pattern is of paramount importance for the success of any face recognition approach. Several techniques have been presented in the literature to achieve this goal, however, techniques exploiting Gabor filters are not only amongst the most popular, but are extremely effective as well.

Gabor filters are capable of deriving multi-orientational information from a face image at several scales, with the derived information being of local nature. The common approach when using Gabor filters for face recognition is to construct a filter bank with filters of different scales and orientations and to filter the given face image with all filters from the bank. Obviously, such an approach results in an explosion of information, as the dimensionality of the input face pattern is increased by a factor equaling the number of filters in the filter bank. The amount of data (in the Gabor face representation) is then commonly reduced to a more manageable size by exploiting various downsampling, feature selection and subspace projection techniques before it is finally fed to a classifier [1].

Unlike other work found in the literature [1], [2], [3], [4] which primarily deals with the problem of effectively reducing the size of the Gabor face representation once this has already been computed, this paper takes a different approach and tries to propose a way of deriving a more compact representation. By presuming that the big increase

Vitomir Štruc, Rok Gajšek and Nikola Pavešić are with the Faculty of Electrical Engineering, University of Ljubljana, Tržaška 25, SI-1000 Ljubljana, Slovenia \{vitomir.struc, rok.gajsek, nikola.pavesic\}@fe.uni-lj.si in the data dimensionality can mainly be linked to the fact that the Gabor filters are not orthogonal, we are capable of devising efficient countermeasures. Rather than using the original filter bank of Gabor filters for the derivation of the Gabor face representation, we propose to employ novel orthogonal filters constructed as a linear combination of the original Gabor filters. As shown in the experimental section, these filters are capable of achieving similar recognition rates than the original ones, but using a far more compact face representation. Since the novel filters are derived from correlation matrices of the original filters by means of principal component analysis, we call them principal Gabor filters.

The rest of the paper is structured as follows. In Section II the theory underlying Gabor filter construction and their use in face recognition systems is briefly reviewed. In Section III the novel principal Gabor filters are introduced and tested in experiments in Section IV. The paper concludes with some final comments in Section V.

\section{GABOR FILTERS FOR FACE RECOGNITION}

This section briefly reviews the basic principles of face recognition using Gabor filters. It commences by introducing the Gabor filters and the Gabor face representation and proceeds by highlighting some characteristics of the filters, which affect the Gabor face representation and consequently the recognition performance of Gabor filter based recognition techniques.

\section{A. Gabor filters}

Gabor filters are among the most popular tools for facial feature extraction. Their use in automatic face recognition system is motivated by two major factors: their computational properties and their biological relevance.

Formally, a 2D Gabor filter in the spatial domain is defined by the following expression [2]:

$$
\psi_{u, v}(x, y)=\frac{f_{u}^{2}}{\pi \gamma \eta} e^{-\left(\frac{f^{2}}{\gamma^{2}} x^{\prime 2}+\frac{f^{2}}{\eta^{2}} y^{\prime 2}\right)} e^{j 2 \pi f_{u} x^{\prime}},
$$

where $x^{\prime}=x \cos \theta_{v}+y \sin \theta_{v}, y^{\prime}=-x \sin \theta_{v}+y \cos \theta_{v}$, and the parameters $f_{u}$ and $\theta_{v}$ are defined as $f_{u}=f_{\max } / 2^{(u / 2)}$ and $\theta_{v}=v \pi / 8$. As we can see, Gabor filters represent Gaussian kernel functions modulated by a complex plane wave whose center frequency and orientation are defined by $f_{u}$ and $\theta_{v}$, respectively. The parameters $\gamma$ and $\eta$ determine the ratio between the center frequency and the size of the Gaussian envelope and, when set to a fixed value, ensure that Gabor filters of different scales and a given orientation 
behave as scaled versions of each other ${ }^{1}$. Commonly the values of $\gamma$ and $\eta$ are set to $\gamma=\eta=\sqrt{2}$. The last parameter $f_{\max }$ denotes the maximum frequency of the filters and is usually set to $f_{\max }=0.25$. By closer examining expression (1) it becomes evident that Gabor filters represent complex filters which combine an even (cosine-type) and an odd (sinetype) [5] part as shown in Fig. 1.

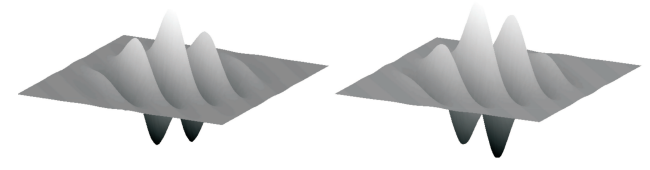

Fig. 1. An example of a Gabor filter: the real (cosine-type) part (left), the imaginary (sine-type) part (right)

When exploited for feature extraction, a filter bank with several filters is usually created and used to extract multiorientational and multi-scale features from the given face image. This filter bank commonly consist of Gabor filters of 5 different scales and 8 orientations, i.e., $u=0,1, \ldots, p-1$ and $v=0,1, \ldots, r-1$, where $p=5$ and $r=8$ [1],[2],[3], [4].

\section{B. Extracting features with Gabor filters}

Let $I(x, y)$ denote a grey-scale face image defined on a grid of size $a \times b$ and let $\psi_{u, v}(x, y)$ represent a Gabor filter determined by the parameters $f_{u}$ and $\theta_{v}$. The filtering operation with the Gabor filter can then be written as follows [1], [2]:

$$
G_{u, v}(x, y)=I(x, y) * \psi_{u, v}(x, y) .
$$

where $G_{u, v}(x, y)$ denotes the complex convolution result which can be decomposed into a real and an imaginary part:

$$
\begin{gathered}
E_{u, v}(x, y)=\operatorname{Re}\left[G_{u, v}(x, y)\right] \\
O_{u, v}(x, y)=\operatorname{Im}\left[G_{u, v}(x, y)\right]
\end{gathered}
$$

Based on the decomposed filtering result both the phase $\phi_{u, v}(x, y)$ as well as the magnitude $A_{u, v}(x, y)$ filter responses can be computed as:

$$
\begin{gathered}
A_{u, v}(x, y)=\sqrt{E_{u, v}^{2}(x, y)+O_{u, v}^{2}(x, y)} \\
\phi_{u, v}(x, y)=\arctan \left(O_{u, v}(x, y) / E_{u, v}(x, y)\right) .
\end{gathered}
$$

Since the computed phase responses vary significantly even for spatial locations only a few pixels apart, Gabor phase features are considered unstable and are usually discarded. The magnitude responses, on the other hand, vary slowly with the spatial position, and are thus the preferred choice when deriving Gabor filter based features.

To derive the Gabor face representation from a given face image $I(x, y)$, the Gabor magnitude responses for the entire filter bank of the 40 Gabor filters are commonly computed first. However, since each of the responses is of the same dimensionality as the input image, this procedure results in an inflation of the original pixel space to 40 times

\footnotetext{
${ }^{1}$ Note that with fixed values of the parameters $\gamma$ and $\eta$ the scale of the Gabor filter is defined by its center frequency $f_{u}$
}

its initial size. To cope with this problem, the magnitude responses are typically downsampled using either a simple rectangular sampling grid or some kind of feature selection scheme. Nevertheless, even after the downsampling, any face representation constructed, for example, by a concatenation of the downsampled magnitude responses, still resides in a high dimensional space. A popular solution to this problem is to use a subspace projection technique, such as principal component analysis or linear discriminant analysis, to further reduce the data's dimensionality.

In the experiments presented in the Section IV of this paper, we use a simple rectangular sampling grid with 256 nodes for the initial dimensionality reduction and linear discriminant analysis for the subspace projection of the feature vector built by concatenating the downsampled magnitude responses.

\section{Properties of Gabor filters}

It is obvious that the mathematical properties of the Gabor filters crucially influence the characteristics and size of the Gabor face representation and thus deserve to be discussed in more detail. A large number of papers dealing with face recognition using Gabor filters emphasize the fact that the filters exhibit properties, such as spatial locality and orientational selectivity, and that they are optimally localized in the space and frequency domains. While these properties are certainly appealing, they might not necessarily be the most important when deriving discriminative and most of all compact representations of a face pattern. Optimal resolution of the filters in both the spatial as well as the frequency domain, for example, is desirable to derive spatially local features of a confined frequency band, but is unfortunately also exactly the reason why the dimensionality of the (notdownsampled) Gabor face representation is that much bigger than the initial size of the input face image.

Another known shortcoming of Gabor filters is the fact that different filters from the filter bank are not orthogonal one to another. The information encoded in the final Gabor face representation is therefore redundant and might affect the recognition accuracy of the classifier relying on the Gabor face representation. In the next Section, we will try to modify the classical Gabor filters in such a way that some of the properties just described are altered and, hence, the new filters are more effective in deriving a compact and discriminative face representation.

\section{PRINCIPAL GABOR FILTERS}

Orthogonality is one of the key elements for many pattern representations. However, as already emphasized in the previous section, Gabor filters do not represent orthogonal filters and hence the information encoded in the filter responses is therefore likely to be redundant. To overcome this problem we propose to orthogonalize the commonly adopted filter bank (featuring filters of 5 scales and 8 orientations and defined by the parameter values given in Section II-A) using principal component analysis (PCA) [6]. 
At this point the question arises how to orthogonalize the filter bank while still (at least partially) preserving other important characteristics such as orientational selectivity and spatial locality. To answer this question let us consider the Gabor filter bank $\mathcal{G}$ defined as $\mathcal{G}=\left\{G\left(f_{u}, \theta_{v}\right)=G_{u, v}: u=\right.$ $0,1, \ldots, p-1 ; v=0,1, \ldots, r-1\}$. For each filter orientation $\theta_{v}$ we can form a complex valued data matrix $\boldsymbol{\Gamma}_{v}$ of size $a b \times p$ whose columns correspond to Gabor filters of different scales rearranged into vector form, i.e, $\boldsymbol{\Gamma}_{v}=\left[\mathbf{g}_{0, v}, \mathbf{g}_{1, v}, \ldots, \mathbf{g}_{p-1, v}\right]$, where $\mathbf{g}_{u, v}$ denotes the column vector corresponding to the Gabor filter $G_{u, v}$. Using the constructed data matrices $\boldsymbol{\Gamma}_{v}$, we can compute a correlation matrix $\boldsymbol{\Sigma}_{v}$ for each filter orientation as follows:

$$
\boldsymbol{\Sigma}_{v}=\boldsymbol{\Gamma}_{v} \boldsymbol{\Gamma}_{v}^{T}
$$

where $T$ stands for the transpose operator.

It can be shown that each correlation matrix $\boldsymbol{\Sigma}_{v}$ has exactly $p$ eigenvectors that correspond to non-zero eigenvalues. These eigenvectors are orthogonal and in appearance quite similar to Gabor filters. The orthogonality of the eigenvectors is a consequence of the symmetry of the correlation matrices, while the appearance of the eigenvectors can be ascribed to the fact that the eigenvectors of a matrix must lie in the span of all data samples (in our case in the span of the Gabor filters in vector form) and are, hence, nothing more than a linear combination of the input data. Fig. 2 shows a visual comparison between the real part of the Gabor filter bank $\mathcal{G}$ and the real part of the eigenvector of all $r$ correlation matrices $\boldsymbol{\Sigma}_{v}$.
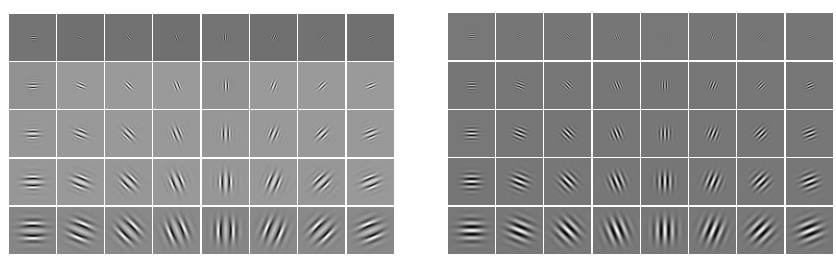

Fig. 2. Visual comparison of the real part of the eigenvectors of the correlation matrices (left) and the real part of the Gabor filter bank (right).

Due to the great similarity between the presented eigenvectors and the original Gabor filters and the fact that the eigenvectors (of each orientation) of the correlation matrices are orthogonal, we propose to employ the unitary eigenvectors rather than the classical filters for the derivation of the Gabor face representation. In analogy with principal component analysis, we will refer to these eigenvectors as principal Gabor filters in the remainder of this paper.

\section{A. Properties of principal Gabor filters}

Recently, Wang et al. [7] proposed to inspect a correlation matrix constructed using a bank of Gabor filters with the goal of selecting such filter parameters that the filters comprising the filter bank would be as uncorrelated as possible. The same methodology can also be used here to visualize the difference between the classical and the principal Gabor filters. To this end, one $(40 \times 40)$ correlation matrix is build for each of the two filter banks. When computing the correlation matrices we arrange the filters first in order of orientation and then scale, thus, the first $8 \times 8$ block in the upper left corner of the matrices presented in Fig. 3 depicts the correlation of the filters of the smallest scale (and different orientations), the next block down the diagonal depicts the correlations for the second filter scale and so forth. We can see that there are still
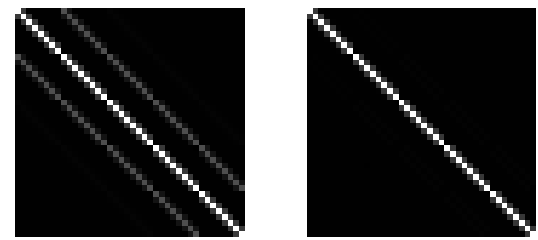

Fig. 3. Visual comparison of the correlation matrices of the classical Gabor filter bank (left) and the principal Gabor filter bank (right).

small correlations between filters of two adjacent orientations for both filter types; however, the principal Gabor filters exhibit no correlations between different filter scales as the classical filters do (i.e., the two lines parallel to the main diagonal are not present in the right image of Fig. 3).

While the orthogonality of the principal Gabor filters might have a positive effect on the (principal) Gabor face representation in terms of compactness, this comes at a price, as the filters are not localized optimally in the spatial nor in the frequency domain anymore. This fact is illustrated in Fig. 4 , where the top row shows the magnitudes of the classical Gabor filters at different scales and the bottom row shows the magnitudes of the principal filters at different scales.
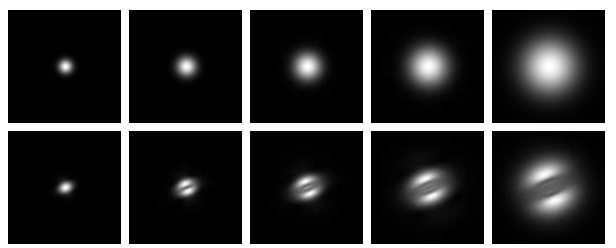

Fig. 4. Comparison of the magnitudes of the two filter types at diff. scales.

Last but not least Fig. 5 shows the coverage of the power spectrum (together with the mirrored channels) of the two filter banks. We can see that, while the classical filters cover the frequency plane evenly, the principal filters give more emphasis to certain frequency bands.
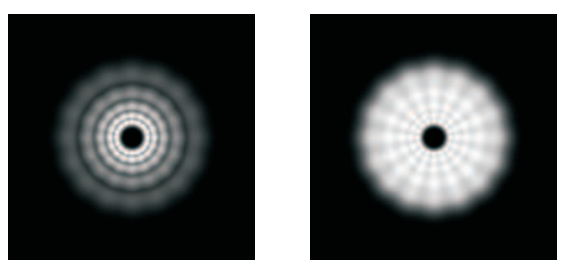

Fig. 5. Comparison of the coverage of the power spectrum: for the principal filter bank (left) and the classical Gabor filter bank (right).

\section{EXPERIMENTAL RESULTS}

\section{A. The Experimental Databases}

To assess the expediency of the proposed principal Gabor filters for face recognition, two popular face databases were 
adopted for the experiments presented in the remainder of this paper - the XM2VTS and YaleB databases [8], [9].

The first, the XM2VTS database, comprises 2360 facial images that correspond to 295 distinct subjects. Each subject in the database is accounted for with 8 facial images, which were taken during four separate recording sessions. The images were captured in front of a uniform background and in controlled illumination conditions. Thus, the variability in the appearance of a given subjects face images is induced mainly by the temporal factor, as the recording sessions were distributed over a period of approximately five months. The images differ in terms of pose, head-rotation, presence/absance of glasses, mustaches and makeup, different hairstyle, etc.

The second, the YaleB face database, contains images of only 10 subjects. These images, however, exhibit large variations in pose and illumination. The database comprises a total of 5760 grey-scale facial images which were taken under 576 different viewing conditions $(9$ poses $\times 64$ illumination conditions). In the experiments presented in this paper, we, however, use only a subset of the database featuring 640 facial images with frontal pose. Some examples of the images from the two databases employed in our experiments are shown in Fig. 6.

Prior to the experiments, a preprocessing procedure was applied to all images of the YaleB and XM2VTS databases. Specifically, the procedure comprised: (i) a rotation and scaling step, which, based on the manually labeled eye coordinates, positioned the eyes at predefined locations; (ii) a cropping step (to a standard size of $128 \times 128$ pixels), which removed all image parts not belonging to the face; and (iii) an image enhancement step in the form of histogram equalization, which improved the contrast of the images [10].

\section{B. Verification experiments on the XM2VTS database}

The experiments on the XM2VTS database were conducted in accordance with the first configuration of the databases experimental protocol [8]. The protocol partitions the subjects of the database into two disjoint groups of clients (200 subjects) and impostors (95 subjects) ${ }^{2}$ and further defines which images from the two groups should be used for training, evaluation and testing. Since the XM2VTS's experimental protocol represents a closed-set protocol, the training images also coincide with the enrollment images of the clients and are also often referred to as development images. These images are exploited to train the given face recognition technique and subsequently to build client models. Images designated for the evaluation phase are used to set an operational decision threshold, while images designated for the test phase are employed solely in the final performance assessment (with a pre-trained recognition technique and a pre-set decision threshold). The details of the employed protocol are shown below:

- number of training images per subject: $n_{t}=3$,

\footnotetext{
${ }^{2}$ Note that clients represent users making a genuine identity claim, while impostors represent users claiming an identity different from their own.
}

- total number of client access trials in the evaluation phase: $n_{c e}=600$ (3 images per subject),

- number of impostor access trials in the evaluation phase: $n_{i e}=40000$ (each of the 25 evaluation impostors claims 200 enrolled identities using 8 different images),

- total number of client access trials in the test phase: $n_{t e}=400$ (2 images per subject),

- number of impostor access trials in the test phase: $n_{t e}=$ 112000 (each of the 70 evaluation impostors claims 200 enrolled identities using 8 different images).

Following the presented experimental protocol, our first series of verification experiments assessed the performance of the principal Gabor filters with respect to the employed number of filter scales. Since the procedure of principal filter creation presented in Section III involves filters of only one orientation at the time, we fixed the angular resolution of our filter bank to the most common value found in the literature and employed eight filter orientations in all of our experiments. We adopted linear discriminant analysis (LDA) for the dimensionality reduction and the nearest neighbor for classification. The results of the experiments are presented in Fig. 7, where the Detection-Trade-Off (DET) curves (which plot the false rejection error rate (FRR) against the false acceptance error rate (FAR) at various values of the decision threshold) are shown. Here, the left graph depicts the performance of the principal Gabor filters in conjunction with LDA, while the right graph depicts the performance of the classical Gabor filters in conjunction with LDA. From the graphs we can see that the verification performance of the principal Gabor filters saturates or at least does not increase significantly from three scales upwards. The performance of the classical Gabor filters, on the other hand, improves with the deployment of more filter scales (for most of the operating points on the DET curves). Fig. 8 shows a more detailed comparison of the best performing configuration of the classical Gabor filters (i.e., 8 orientations and 5 scales) and the principal Gabor filters (i.e., 8 orientations and 3 filter scales) on the evaluation image set. For baseline comparisons the performance of the popular Fisherface technique (denoted as LDA) [11] is also given in the graph.

The results clearly show that both filter types improve upon the performance of the raw image data, while the direct comparison of the principal and classical filters suggests that the former perform better at operating points corresponding to low false acceptance rates and the classical Gabor filters outperform the principal ones at operating points corresponding to higher values of the false acceptance rate.

Before we make any final conclusions, let us further examine the performance of both filter types using the test image set. To this end, we first fix the decision threshold on the evaluation image set to the equal error operating point ${ }^{3}$ and then perform verification experiments on the test image set with this pre-set decision threshold. The results of this

\footnotetext{
${ }^{3}$ The equal error operating point is defined as the point on the DET curve where the values of the false acceptance error rate and the false rejection error rate are equal.
} 

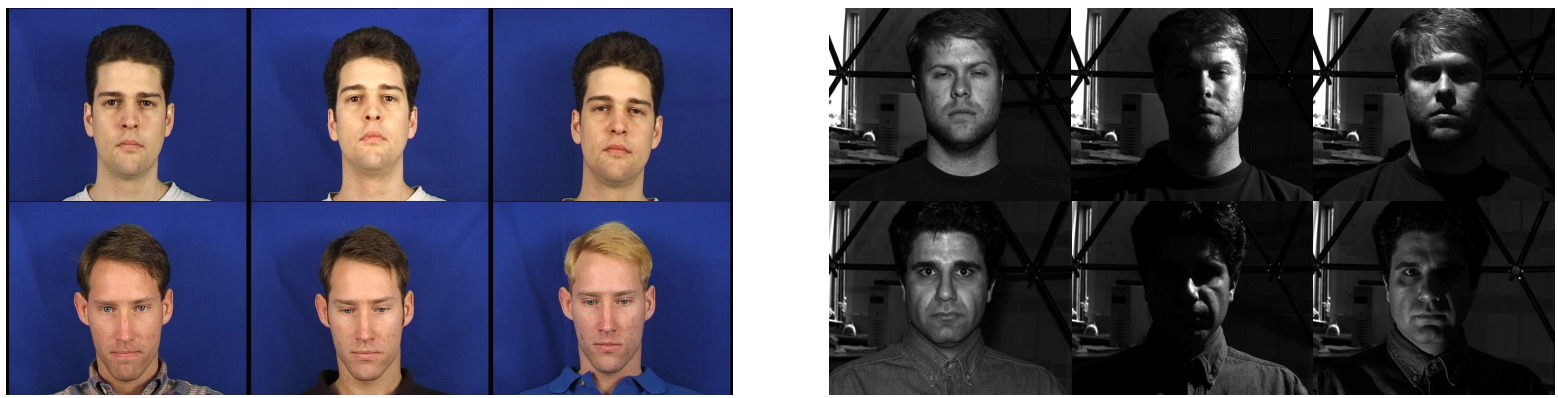

Fig. 6. Sample images from the employed databases: examples from the XM2VTS database (left image block), examples from the YaleB database (right image block).
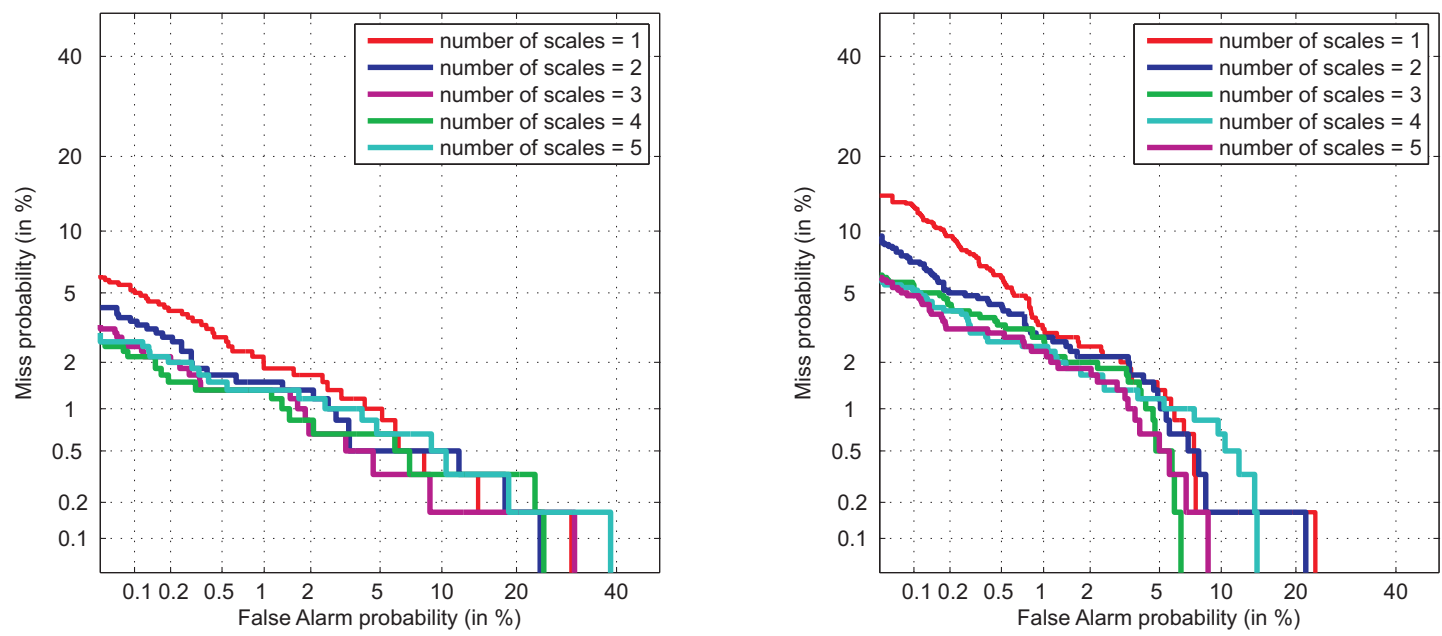

Fig. 7. DET curves generated during the evaluation experiments: principal Gabor filters + LDA (left), Gabor filters + LDA (right).

TABLE I

THE VALUES OF FAR, FRR AND HTER (ON THE TEST SET) FOR DIFFERENT CONFIGURATIONS OF THE CLASSICAL AND PRINCIPAL GABOR FILTERS AS WELL AS FOR THE FISHERFACE TECHNIQUE.

\begin{tabular}{|c|c|c|c|c|c|c|c|c|c|c|c|}
\hline Method & LDA & p1 & p2 & p3 & p4 & p5 & g1 & g2 & g3 & g4 & g5 \\
\hline \hline FAR (\%) & 4.04 & 1.54 & 1.17 & 1.18 & 1.15 & 1.46 & 0.83 & 1.19 & 1.04 & 0.98 & 0.99 \\
FRR (\%) & 3.25 & 1.75 & 0.75 & 0.50 & 1.00 & 1.00 & 2.25 & 1.50 & 1.25 & 1.00 & 0.75 \\
HTER (\%) & 3.65 & 1.65 & 0.96 & 0.84 & 1.08 & 1.23 & 1.54 & 1.34 & 1.15 & 0.99 & 0.87 \\
\hline
\end{tabular}

part of our assessment are presented in Table IV-B. Here, we again measure the performance in terms of the FAR and FRR and, furthermore, provide the values of the half total error rate (HTER), which is defined as the average value of the FAR and FRR. It should be noted that the letter "p" in Table IV-B denotes the principal filters, while "g" stands for the classical Gabor filters. In all cases the number next to the letter stands for the number of employed filter scales.

Similar to the evaluation experiments we again notice that the performance of the principal filters peaks when three filter scales are employed for filtering. The best performance with the classical filters is again observed with five filter scales. While the difference in the HTERs of the top performing filter configurations of both filter types at the tested operating point is statistically not necessarily significant, another observation is far more important. The classical approach of Gabor filter deployment requires 40 filters for its top performance, the approach with the principal filters, on the other hand, requires only 24 filters for the same HTER. Moreover, as the same downsampling factor was used for both filter types, the feature vector derived using the principal filters (prior to dimensionality reduction with LDA) is less dimensional than the one obtained with the classical approach. Hence, the verification performance could be further improved by using a smaller downsampling factor for the (principal Gabor) filtered images.

\section{Identification experiments on the YaleB database}

For the identification experiments on the YaleB database we partitioned the database into five subsets according to the extremity in the illumination conditions present during the image acquisition stage. Thus, the first subset contained images captured under relatively "good" illumination, the second images captured in slightly worse illumination conditions and so forth. Even though the database contains some corrupted images, these were not removed from the subsets. 
TABLE II

THE RANK ONE RECOGNITION RATES (IN \%) FOR THE YALEB DATABASE FOR DIFFERENT CONFIGURATIONS OF THE CLASSICAL AND PRINCIPAL GABOR FILTERS AS WELl AS FOR THE FISHERFACE TECHNIQUE.

\begin{tabular}{|c|c|c|c|c|c|c|c|c|c|c|c|}
\hline Method & LDA & p1 & p2 & p3 & p4 & p5 & g1 & g2 & g3 & g4 & g5 \\
\hline \hline subset 2 & 100 & 100 & 100 & 100 & 100 & 100 & 100 & 100 & 100 & 100 & 100 \\
subset 3 & 100 & 100 & 100 & 100 & 100 & 100 & 100 & 100 & 100 & 100 & 100 \\
subset 4 & 63.6 & 95.0 & 94.3 & 96.4 & 94.3 & 95.0 & 89.3 & 92.1 & 92.1 & 92.1 & 92.1 \\
subset 5 & 14.2 & 97.4 & 97.9 & 99.0 & 97.4 & 96.8 & 91.6 & 94.7 & 94.7 & 96.3 & 95.3 \\
\hline
\end{tabular}

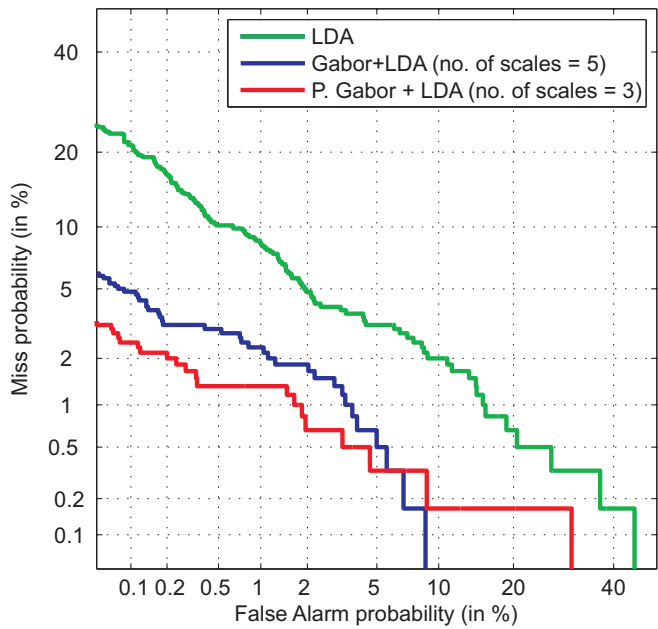

Fig. 8. Comparison of best performing filter configurations for the Gabor and principal Gabor filters and the Fisherface technique

For the training set the first image subset featuring 7 images per subject was adopted, while the remaining image sets were employed for testing. It should be noted that such an experimental configuration is also in accordance with real-life settings, as the enrollment process can always be supervised, whereas the operational conditions are typically unknown in advance. A more detailed description of the protocol is given below:

- number of training images per subject: $n_{t}=7$,

- number of ident. attempts with subset 2: $n_{s 2}=120$,

- number of ident. attempts with subset 3: $n_{s 3}=120$,

- number of ident. attempts with subset 4: $n_{s 4}=140$,

- number of ident. attempts with subset 5: $n_{s 5}=190$.

Similarly to the experiments presented in the previous section, LDA was exploited as the dimensionality reduction technique, and the nearest neighbor classifier with the cosine similarity measure was adopted for the classification. The length of the feature vector was set to its maximum value (i.e., 9 for the YaleB database). The results of the experiments are presented in Table IV-B.

The results of this series of experiments again suggest that only three filter scales are needed to achieve the best performance with the proposed principal Gabor filters. Using more than three filter scales actually led to a decreased recognition performance. Interestingly, the classical approach with four filter scales performed a bit better than the commonly used variant with five filter scales. All in all we can conclude that using principal Gabor filters instead of the classical Gabor filters results in a similar recognition performance; however, with a significant improvement in the computational complexity, as only 24 ( 3 scales and 8 orientations) instead of 40 filters are needed for computing the Gabor face representation.

\section{CONCLUSION}

We have presented novel image filters derived from the popular Gabor filters, which can be used for feature extraction in the field of face recognition. Unlike the classical Gabor filters, the proposed principal Gabor filters are orthogonal and, hence, are capable of deriving face representation where the encoded information is not redundant. Experimental results obtained in a series of verification and identification experiments suggest that the new filters result in a similar performance as the classical Gabor filters with a significantly reduced computational complexity. Our future research with respect to principal Gabor filters will be focused on assessing the performance of the filters on a larger database and deploying alternative techniques for filter decorrelation, such as independent component analysis.

\section{REFERENCES}

[1] C. Liu, "Capitalize on dimensionality increasing techniques for improving face recognition grand challenge performance," TPAMI, vol. 11, no. 4, pp. 467-476, 2002.

[2] L. Shen, L. Bai, and M. Fairhurst, "Gabor wavelets and general discriminant analysis for face identification and verification," Image and Vision Computing, vol. 25, no. 5, pp. 553-563, 2007.

[3] C. Liu and H. Wechsler, "Gabor feature based classification using the enhanced fisher linear discriminant model for face recognition," TPAMI, vol. 28, no. 5, pp. 725-737, 2006.

[4] V. Štruc and N. Pavešić, "Gabor-based kernel partial-least-squares discrimination features for face recognition," Informatica, vol. 20, no. 1, pp. 115-138, 2009.

[5] M. Lades, J. Vorbruggen, J. Buhmann, J. Lange, C. von der Malsburg, R. Wurtz, and W. Konen, "Distortion invariant object recognition in the dynamic link architectrue," IEEE Transactions on Computers, vol. 42, no. 3, pp. 300-311, 1993.

[6] M. Turk and A. Pentland, "Eigenfaces for recognition," Journal of Cognitive Neurosicence, vol. 3, no. 1, pp. 71-86, 1991.

[7] L. Wang, Y.Li, C. Wang, and H. Zhang, "2d gaborface representation method for face recognition with ensemble and multichannel model," Image and Vision Computing, vol. 26, no. 6, pp. 820-828, 2008.

[8] K. Messer, J. Matas, J. Kittler, J. Luettin, and G. Maitre, "Xm2vtsdb: the extended m2vts database," in Proceedings of AVBPA'99, Washington D.C., USA, March 1999, pp. 72-77.

[9] P. B. A.S. Georhiades and D. Kriegman, "From few to many: Illumination cone models for face recognition under variable lightning and pose," TPAMI, vol. 23, no. 6, pp. 643-660, 2001.

[10] V. Štruc, F. Mihelič, and N. Pavešić, "Face authentication using a hybrid approach," J. of Electronic Imaging, vol. 17, no. 1, pp. 1-11, 2008.

[11] P. Belhumeur, J. Hespanha, and D. Kriegman, "Eigenfaces vs. fisherfaces: Recognition using class specific linear projection," in Proceedings of the 4th ECCV, Cambridge, UK, April 1996, pp. 45-58. 